\title{
STM-SEM and impedance characterization of columnar structured gold electrodes
}

\author{
M.M. Gómez ${ }^{\text {a }}$, L. Vázquez ${ }^{\text {b }}$, R.C. Salvarezza ${ }^{\text {a }}$, J.M. Vara ${ }^{\text {a }}$ and A.J. Arvia ${ }^{\text {a* }}$ \\ "Departamento de Quimica-Física Aplicada, C-II, Facultad de Ciencias, Universidad Autónoma de Madrid, \\ 28049 Madrid (Spain) \\ ${ }^{b}$ Instituto de Ciencias de Materiales, sede B, CSIC, Departamento Fisica Aplicada, C-XII, \\ Facultad de Ciencias Universidad Autónoma de Madrid, 28049 Madrid (Spain)
}

(Received 23 April 1991; in revised form 28 June 1991)

\begin{abstract}
The impedance spectra of thin gold electrodeposits with $10^{-6}-10^{-5} \mathrm{~cm}$ average columnar radius and $4 \times 10^{-6}-3 \times 10^{-4} \mathrm{~cm}$ average height have been measured in acid solutions. Frequency dispersion has been observed for film thicknesses greater than $3 \times 10^{-5} \mathrm{~cm}$ and electrolyte resistivities larger than $300 \Omega$ $\mathrm{cm}^{-1}$. STM-SEM observations reveal a self-affine surface structure with a size distribution of pores ranging from $10^{-6}$ to $10^{-4} \mathrm{~cm}$ between the columnar elements. In diluted solutions the contribution of the smallest pores to the sampled electrode area decreases as $\omega$ increases explaining the observed frequency dispersion. This result confirms that self-affine surfaces exhibit a fractional-power-law impedance. However, no simple correlation was found between the $\alpha$ value and the $D$ value of the self-affine surface measured by an independent method. The present results also show that the electrolyte resistivity plays a certain role in determining the $\alpha$ value.
\end{abstract}

\section{INTRODUCTION}

The impedance, $Z(\omega)$, of rough and porous electrodes has been the subject of recent work carried out to determine the properties of real surfaces, particularly the roughness characteristics, in terms of complex equivalent circuits [1-4]. Measurements of this type seem to be very promising as a possible way to study euclidean and fractal rough structures [4].

The impedance spectra of rough metal electrodes i.e. the $Z(\omega)$ vs, $\omega$ plot can be usually described by the empirical relationship:

$Z(\omega)=A(i \omega)^{-\alpha}$

* Visiting Professor from INIFTA, La Plata, Argentina. 
where $A$ is a frequency-independent parameter, $i=(-1)^{1 / 2}, \omega$ is the angular velocity ( $\omega=2 \pi f, f$, the frequency) and $\alpha$ is a number between 0 and 1 . In the absence of faradaic processes, $Z(\omega)$ is given by $(i \omega C)^{-1}$, so the $\log \omega C$ vs. $\log \omega$ plot gives the $\alpha$ value. For smooth surfaces $\alpha$ is equal to 1 , and for long pore structured electrodes $\alpha$ becomes equal to 0.5 [5]. This means that $\alpha$ appears to be a structure dependent parameter. Accordingly, attempts were made to relate $\alpha$ to $D$, the fractal dimension of the metal electrode surfaces. However, for an electrode characterized by a certain value of $\alpha, D$ depends on the chosen model [6-9], as explained in ref. 4. In spite of this, interesting information on the structure of rough metal interfaces can be obtained from an analysis of the frequency dispersion [10]. It should be noted that most of the works published on this matter are concerned with mathematical fractals [6], dispersed metal powders [10], large dendritic [11] and highly ramified fractal blocking electrodes [12] with dimensions ranging from $10^{-3}$ to $1 \mathrm{~cm}$. Conversely, little is known about the impedance spectrum of rough columnar metal films, i.e., those films with an average thickness in the range $10^{-5}$ to $10^{-3} \mathrm{~cm}$, grown from the vapor phase or by electrodeposition from metal oxides. The surface of these films has been described either as self-affine [13] or self-similar fractals [14] although the nature of the elements which exhibit scaling behaviour remains unclear.

Here we report on the surface of electrodispersed columnar gold electrodes (EDAuE) [15-17] with controlled surface roughness by using scanning tunneling microscopy (STM) and scanning electron microscopy (SEM), and correlate the data obtained by these techniques to the impedance spectrum of the films. The influence of thin metal film parameters such as film thickness, column and pore size as well as the electrolyte composition and resistivity on the frequency dependence of the impedance spectrum are investigated in order to establish the origin of the frequency dispersion in these systems.

\section{EXPERIMENTAL}

\section{Electrode preparation}

The in-situ preparation of the EDAuE's has been extensively described elsewhere [15-17]. Briefly, a polycrystalline $\mathrm{Au}$ wire electrode immersed in $0.5 \mathrm{M} \mathrm{H}_{2} \mathrm{SO}_{4}$ at room temperature was anodized at $2.6 \mathrm{~V}$ (SHE) for a preset time to accumulate a certain amount of hydrous gold oxide depending on the duration of the anodization treatment. The hydrous gold oxide layer was subsequently electroreduced through a potential sweep run at $0.1 \mathrm{~V} / \mathrm{s}$ from $1.65 \mathrm{~V}$ to $0 \mathrm{~V}$. The resulting EDAuE had an average thickness in the range between $4 \times 10^{-6}$ to $3 \times 10^{-4} \mathrm{~cm}$ depending on the amount of the hydrous gold oxide accumulated as a layer on the gold substrate. The active surface area of the EDAuE's (ARA) was voltammetrically determined in $1 \mathrm{M}$ $\mathrm{H}_{2} \mathrm{SO}_{4}$ through the ratio between the $\mathrm{O}$-adatom monolayer electrodesorption charge

and the O-adatom monolayer electrodesorption; charge density taken as 0.42 $\mathrm{mC} / \mathrm{cm}^{2}$ [17]. The corresponding roughness factor, $R$, was defined as the quotient 
of ARA and the initial active area (IAA) evaluated before applying the electrochemical roughening treatment. This means that $R=\mathrm{ARA} / \mathrm{IAA}$, using the O-atom as the yardstick.

\section{Impedance measurements}

Impedance measurements were made in a conventional three electrode cell with a large area $\mathrm{Au}$ counterelectrode, a $\mathrm{Hg} / \mathrm{Hg}_{2} \mathrm{SO}_{4}$ reference electrode and the EDAuE working electrode immersed in either $x \mathrm{M} \mathrm{H}_{2} \mathrm{SO}_{4}$ or $x \mathrm{M} \mathrm{HClO}_{4}\left(10^{-4}<x<1\right)$. The specific resistivities, $\rho$, of these solutions were in the range $1 \times 10^{4}-5 \Omega \mathrm{cm}$ Impedance measurements were made by using a conventional electrochemical impedance bridge which gives accurate data in the dilute acid solutions. The impedance spectra were obtained at $0.30 \mathrm{~V}$ (SHE), a potential region where no faradaic reactions could be detected, so that the electrode approaches the ideal conditions of a metal blocking electrode. The working frequency range was selected as $10^{2}<f<10^{4} \mathrm{~Hz}$ in order to avoid any electronic artifacts. Accordingly, no influence of the electrode position in the electrochemical cell on the $\alpha$ value was detected. All impedance measurements were made by applying to the working electrode a small signal amplitude of $7 \mathrm{mV}$.

The value of $R$ for the EDAuE's immersed in acid solutions decays slowly after the first $10 \mathrm{~min}$. This process which results in a decrease of the active surface area, involves times in the range of $10^{4} \mathrm{~s}$ [17]. The impedance measurements were made at different times in the slow decay regime $(t>10 \mathrm{~min})$. As the impedance measurement itself involved a relatively short time, the change in $R$ taking place during the measurements could be neglected. This approximation was justified by the fact that no hysteresis in the capacitance values $(C)$ during the impedance measurements could be observed.

\section{STM measurements}

STM measurements were made using a piezo tube STM microscope operating in air. Cut platinum wires ( $0.2 \mathrm{~mm}$ dia.) were used as tips. Measurements were made using a constant tunnel current of 1-2 nA with a tunnel voltage of $0.1 \mathrm{~V}$ (tip +). Data were acquired with an automated work-station and stored as digitized images. The latter are presented as grey scale representations.

\section{RESULTS}

\section{SEM and STM measurements}

SEM micrographs of the cross section of EDAuE's $(R=80)$ are shown in Fig. 1. The film thickness lies between $1 \times 10^{-4}-1.5 \times 10^{-4} \mathrm{~cm}$ (Fig. 1a). At this magnification small height differences in the profile along practically $5 \times 10^{-4} \mathrm{~cm}$ are observed suggesting the self-affine nature of the electrode surface [14]. A detailed 

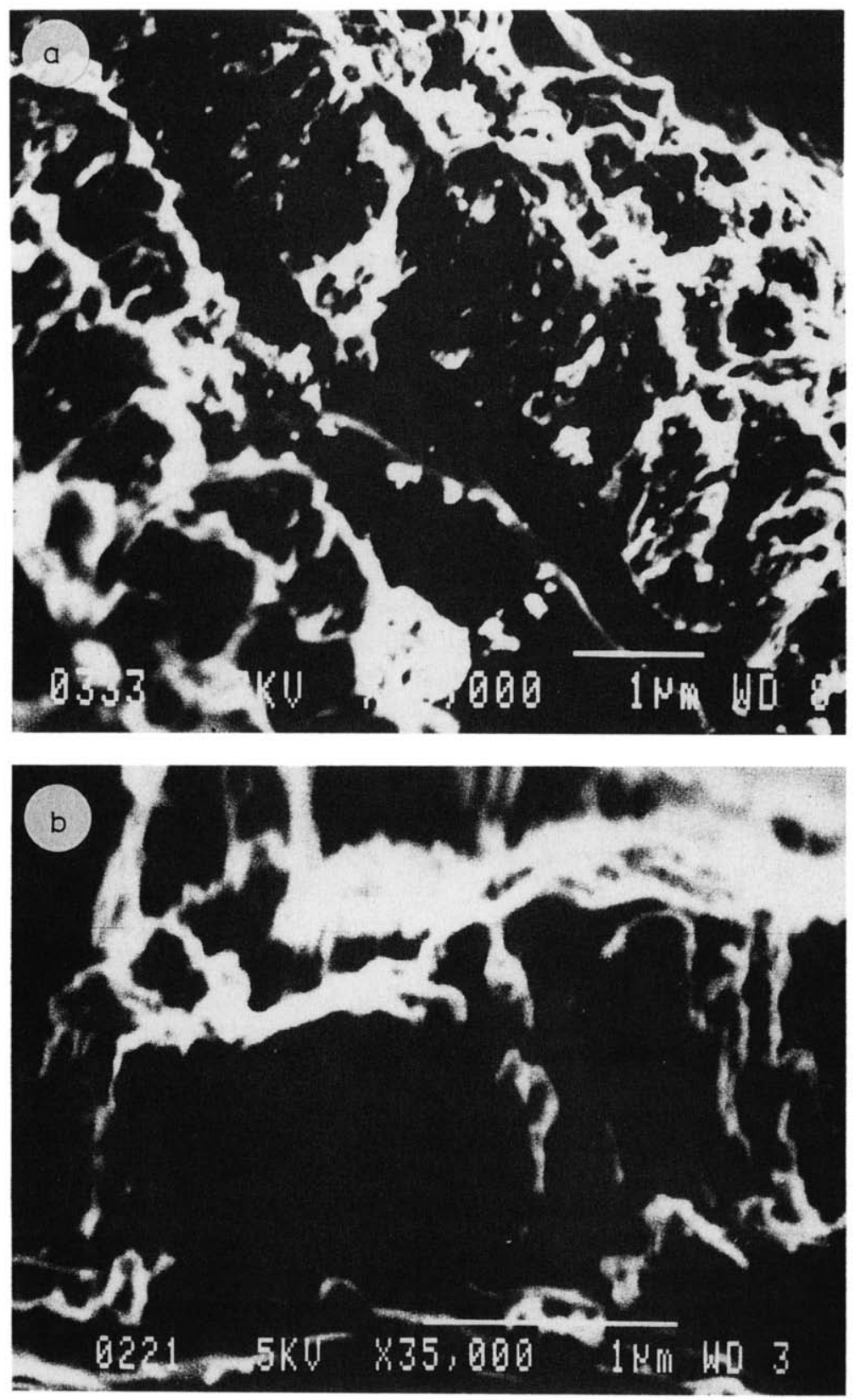

Fig. 1. Cross-sectional SEM micrographs at various magnifications of EDAuE's showing the columnar structure. 

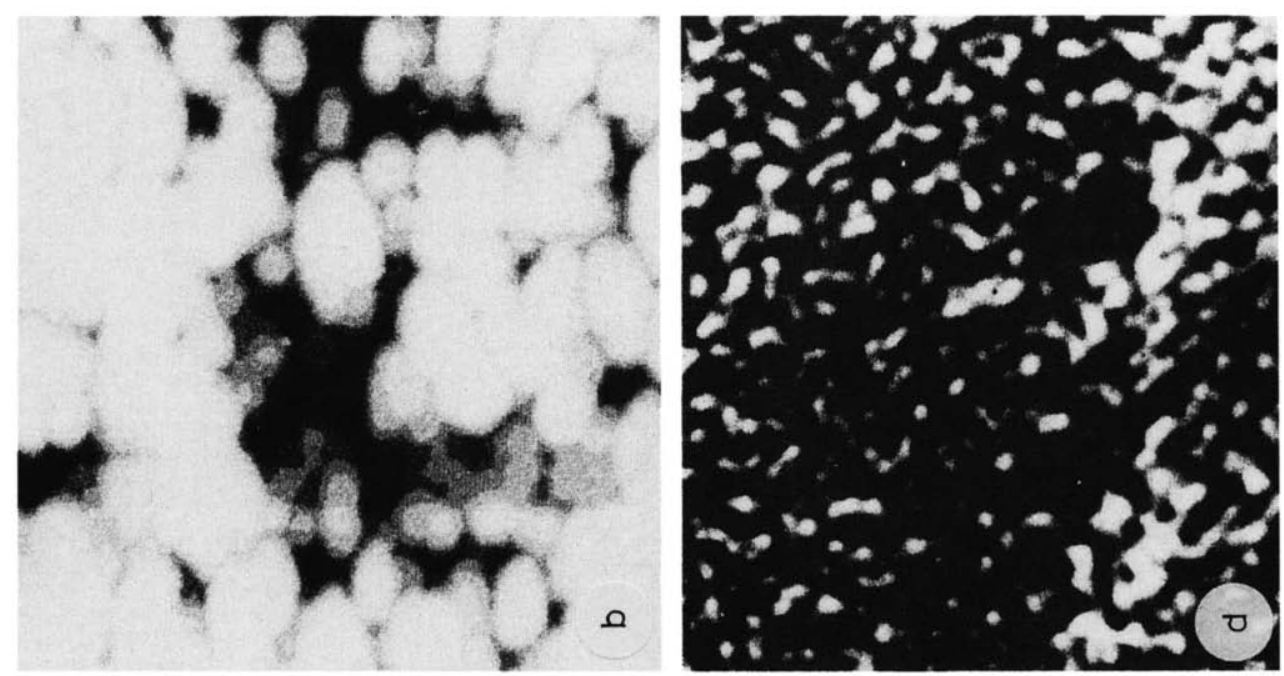

告
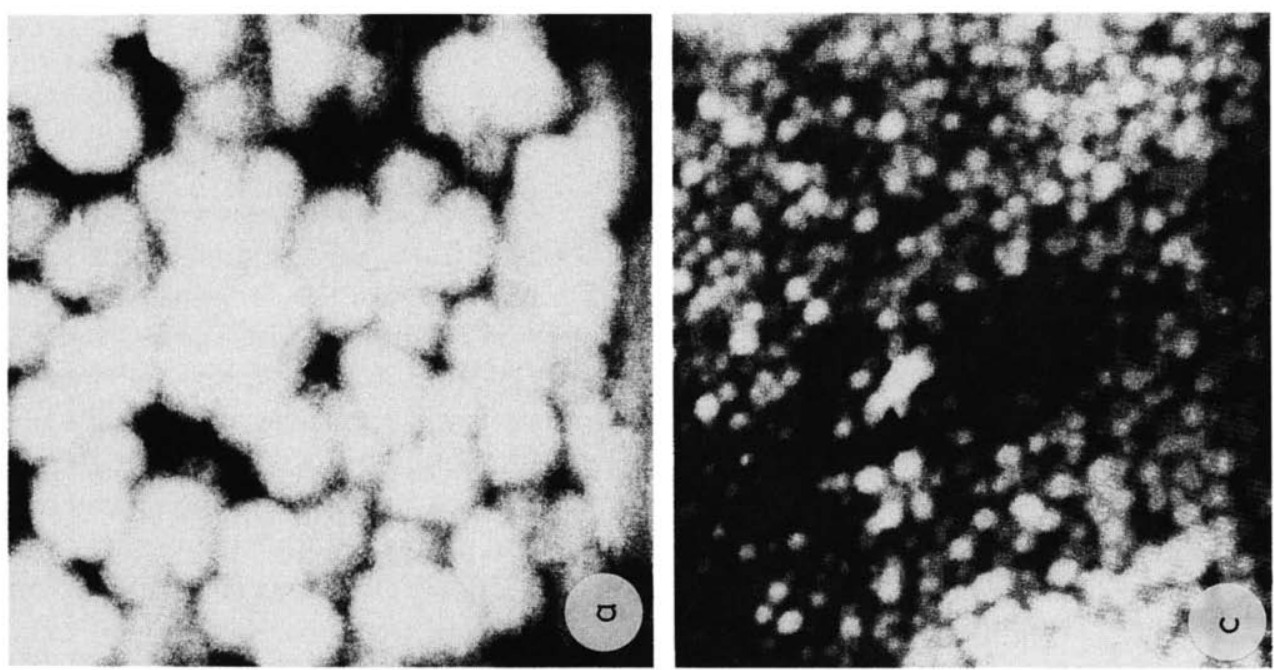

जै

뇽

象

혼

은

蔽

焉

ธิ

롭

政

듬

\&

点

ธิธี ํㅗ

항

要

需豆

$\sum \sum_{1}$

을

离 


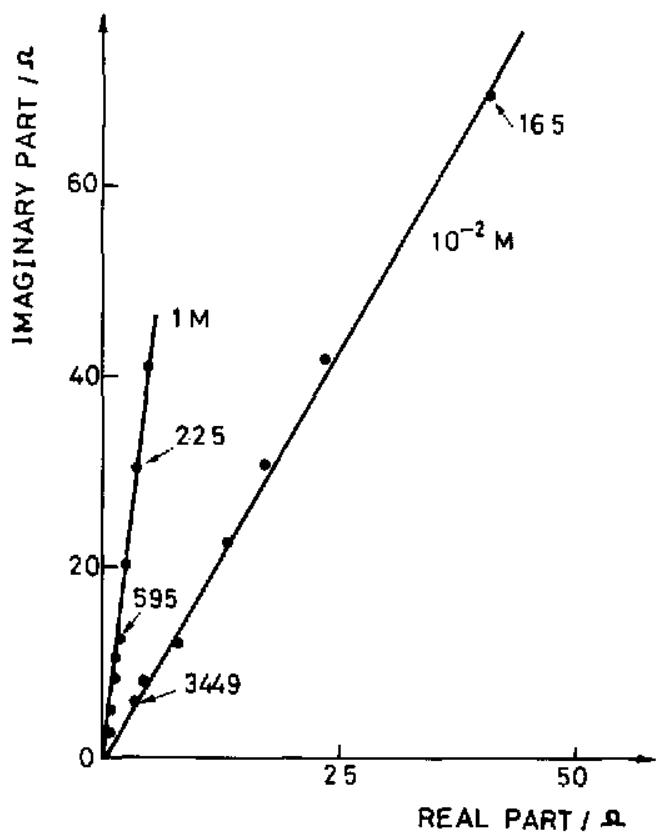

Fig. 3. Impedance spectra of $\mathrm{EDAuE}(R=39)$ obtained in $\mathrm{H}_{2} \mathrm{SO}_{4}$ at various concentrations. The arrows indicate of various $\omega$ values in $\mathrm{Hz}$.

analysis at higher magnifications (Fig. 1b) shows poorly resolved irregular elongated grains and a void network as expected for a columnar microstructure [13].

A series of micrographs of the EDAuE surface $(R=80)$ taken in the $\mu \mathrm{m}$ (SEM)-nm (STM) range is shown in Fig. 2. The surface looks similar at all scale lengths with ramified pores between the small rounded top of the columns. It should be noted that a similar pore shape is observed by STM and SEM indicating that tip geometry has no influence on the STM images. The tops of the columns are smooth and without defects, i.e. steps up to a resolution of $1 \mathrm{~nm}$. From the micrographs the average column top size was estimated to be $30 \mathrm{~nm}$, and pore sizes between $20 \mathrm{~nm}$ and $2000 \mathrm{~nm}$. We have also grown gold films of various thicknesses. The changes in film thickness from $1 \times 10^{-5} \mathrm{~cm}$ to $3 \times 10^{-4} \mathrm{~cm}$ results in the increase of the average size of column tops from $30 \mathrm{~nm}$ to $60 \mathrm{~nm}$. Accordingly, we also observe an increase in the pore size.

\section{Electrochemical measurements}

Typical impedance spectra obtained for EDAuE $(R \equiv 30)$ in $1 \mathrm{M} \mathrm{H}_{2} \mathrm{SO}_{4}$ and $10^{-2} \mathrm{M} \mathrm{H}_{2} \mathrm{SO}_{4}$ after subtraction of the constant real part are shown in Fig. 3. The $\log \omega C$ vs. $\log \omega$ plots exhibit straight line relationships, the slopes of which $(\alpha)$ change from 0.94 to 0.76 when the electrolyte concentration (c) decreases from $1 \mathrm{M}$ 


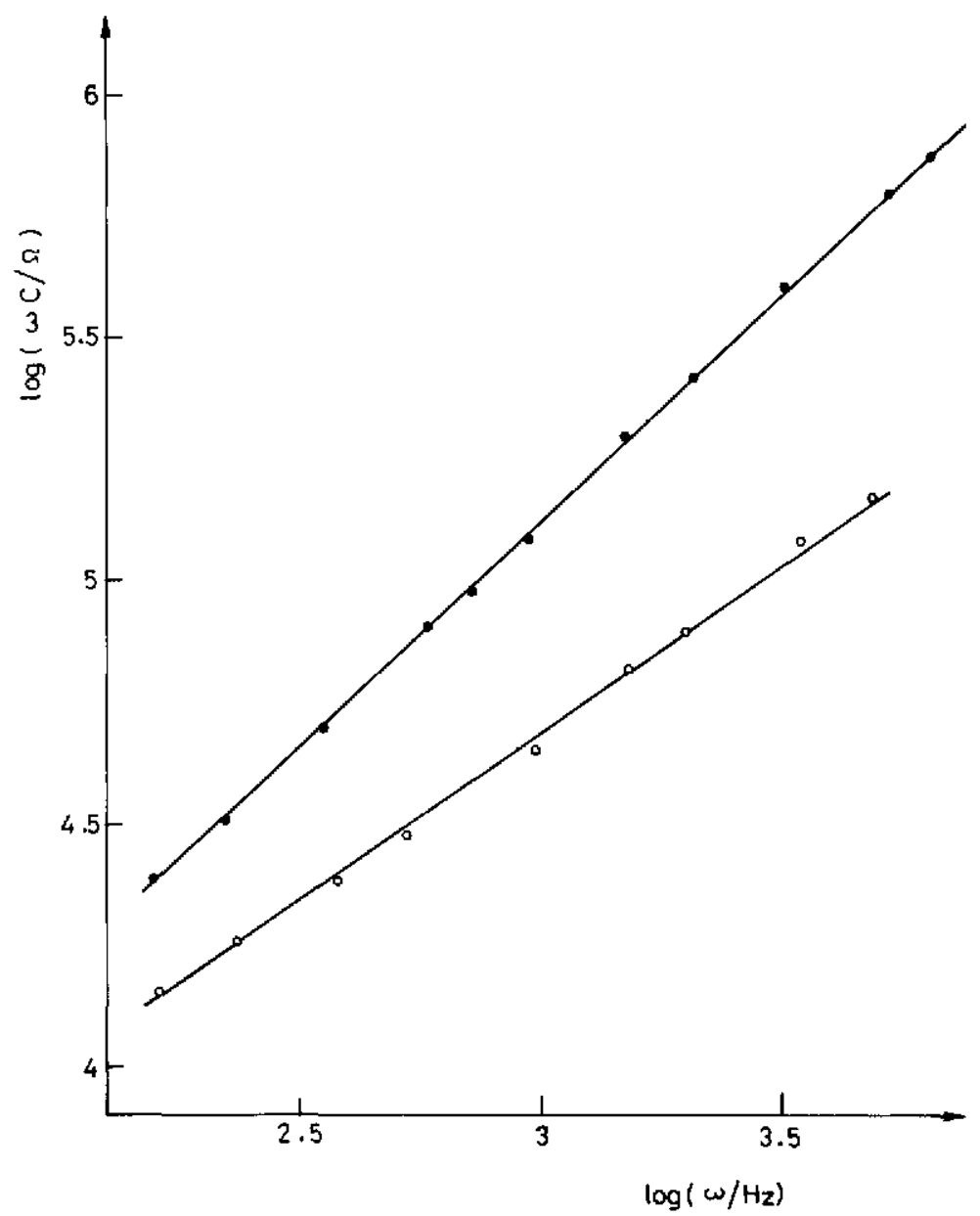

Fig. 4. Plots of $\log \omega C$ vs. $\log \omega$ for the results shown in Fig. 1. (๑) $1 \mathrm{M} \mathrm{H}_{2} \mathrm{SO}_{4}$, (O) $1 \times 10^{-2} \mathrm{M} \mathrm{H}_{2} \mathrm{SO}_{4}$.

to $10^{-2} \mathrm{M}$ (Fig. 4). This effect depends also on the value of $R$. For the limiting case of a smooth Au electrode $(R=1)$ or for EDAuE's showing small $R$ values $(R=4)$, $\alpha$ decreases slightly with $c$ (Table 1 ). Conversely, for the EDAuE's with large $R$ values $(R>30)$ the value of $\alpha$ decreases markedly from 0.95 to 0.76 as $c$ moves from $1 \mathrm{M}$ to $10^{-2} \mathrm{M}$ and then decreases slightly to reach 0.68 for $c=10^{-4} \mathrm{M}$ (Table 1). It should be noted that for $c=1 \mathrm{M}$ all the electrodes show an apparently common value of $\alpha(\alpha=0.95 \pm 0.02)$. The addition of $\mathrm{Na}_{2} \mathrm{SO}_{4}$ to $10^{-3} \mathrm{M} \mathrm{H}_{2} \mathrm{SO}_{4}$ to reach a similar ion concentration to the $1 \mathrm{M}$ solution, yields a value of $\alpha=0.93$. This result becomes independent of the value of $R$. The relationship between $\alpha$ and $c$ becomes nearly independent of the nature of the electrolyte solution, although the values of $\alpha$ obtained in $\mathrm{HClO}_{4}$ solutions are slightly different from those found in 
TABLE 1

Average values of $\alpha$ for EDAuE's in $\mathrm{H}_{2} \mathrm{SO}_{4}$ solutions at various $R$ values

\begin{tabular}{lll}
\hline$c / \mathrm{mol} \mathrm{L}^{-1}$ & $\alpha_{R<6}$ & $\alpha_{R>20}$ \\
\hline 1 & $0.95 \pm 0.02$ & $0.95 \pm 0.02$ \\
$10^{-1}$ & $0.93 \pm 0.02$ & $0.91 \pm 0.02$ \\
$10^{-2}$ & $0.93 \pm 0.02$ & $0.76 \pm 0.04$ \\
$10^{-3}$ & $0.91 \pm 0.02$ & $0.74 \pm 0.04$ \\
$10^{-4}$ & $0.92^{2}$ & $0.68^{*}$ \\
\hline
\end{tabular}

These values exhibit a greater error than those obtained in the more concentrated solutions.

TABLE 2

Values of $\alpha$ for EDAuE in $\mathrm{H}_{2} \mathrm{SO}_{4}$ and $\mathrm{HClO}_{4}$ at various concentrations

\begin{tabular}{ll}
\hline Solution & $\alpha(R=30)$ \\
\hline $1 \mathrm{M} \mathrm{H}_{2} \mathrm{SO}_{4}$ & $0.95 \pm 0.02$ \\
$10^{-3} \mathrm{M} \mathrm{H}_{2} \mathrm{SO}_{4}$ & $0.74 \pm 0.04$ \\
$1 \mathrm{M} \mathrm{HClO}_{4}$ & 0.97 \\
$10^{-3} \mathrm{M} \mathrm{HClO}_{4}$ & 0.66 \\
\hline
\end{tabular}

$\mathrm{H}_{2} \mathrm{SO}_{4}$ solutions (Table 2). These differences are principally due to differences in the corresponding resistivity values.

Two effects might account for the complicated surface impedance of these electrodes. The first is related to the surface roughness and the second to the chemical surface heterogeneity [18]. In order to establish whether the observed frequency dispersion is connected to surface roughness, runs were made by using EDAuE's of differing $R$ values. The increase in $R$ from 1 to 130 shows no appreciable effect on the $\alpha$ value determined in $1 \mathrm{M} \mathrm{H}_{2} \mathrm{SO}_{4}$ (Table 3) whereas a decrease of the value of $\alpha$ from 0.93 to 0.74 in $10^{-3} \mathrm{M} \mathrm{H}_{2} \mathrm{SO}_{4}$ for EDAuE's with $R>20$ (Fig. 5) is observed. On the other hand, the current/potential profiles of the EDAuE's recorded at $0.1 \mathrm{~V} / \mathrm{s}$ in $1 \mathrm{M} \mathrm{H}_{2} \mathrm{SO}_{4}$ show no differences in the shapes and potentials of the O-electrosorption current peaks, as $R$ increases from 4 to 80 . In all cases the shape of the voltammograms resembles closely that corresponding to a

TABLE 3

Values of $\alpha$ for various $R$ in $1 \mathrm{M} \mathrm{H}_{2} \mathrm{SO}_{4}$

\begin{tabular}{rl}
\hline $\mathbf{R}$ & $\alpha$ \\
\hline 1 & $0.95 \pm 0.02$ \\
13 & $0.97 \pm 0.02$ \\
32 & $0.97 \pm 0.02$ \\
50 & $0.93 \pm 0.02$ \\
132 & $0.95 \pm 0.02$ \\
\hline
\end{tabular}




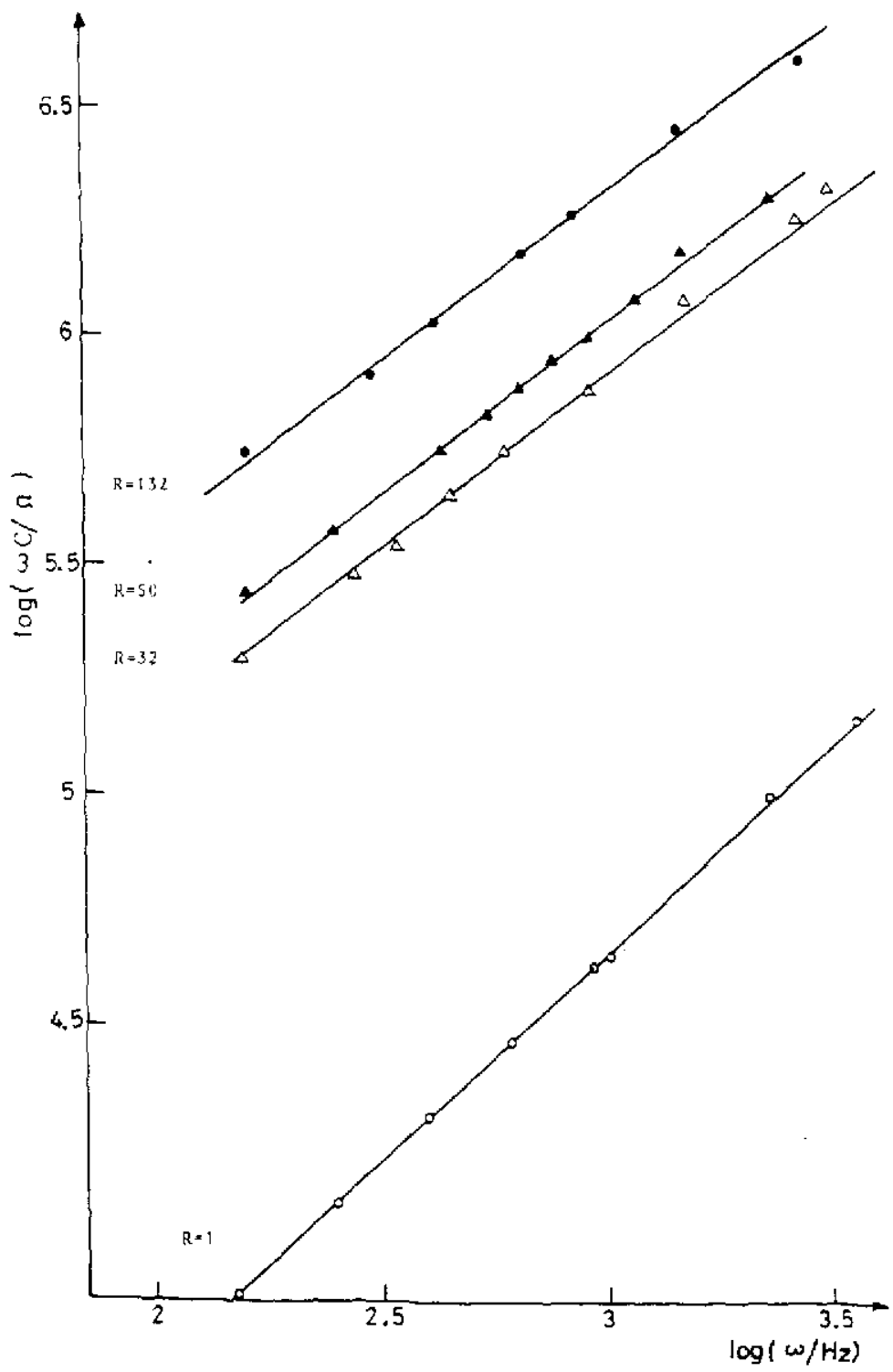

Fig. 5. Plots of $\log \omega C /$ vs. $\log \omega$ for EDAuE's with various $R$ values in $1 \times 10^{-3} \mathrm{M} \mathrm{H}_{2} \mathrm{SO}_{4} ;(0) R=1$,

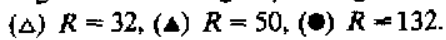

polycrystalline gold electrode. Then, a change in the chemistry of the surface with $R$ can be discarded as the origin of the complicated surface impedance which can be attributed to surface roughness.

The value of $R$ for an EDAuE ( $h \equiv 5 \times 10^{-5} \mathrm{~cm}$ ) decays with time by ageing in 1 $\mathrm{M} \mathrm{H}_{2} \mathrm{SO}_{4}$ (Fig. 6). Nevertheless, it should be noted that despite the fact that $R$ has 

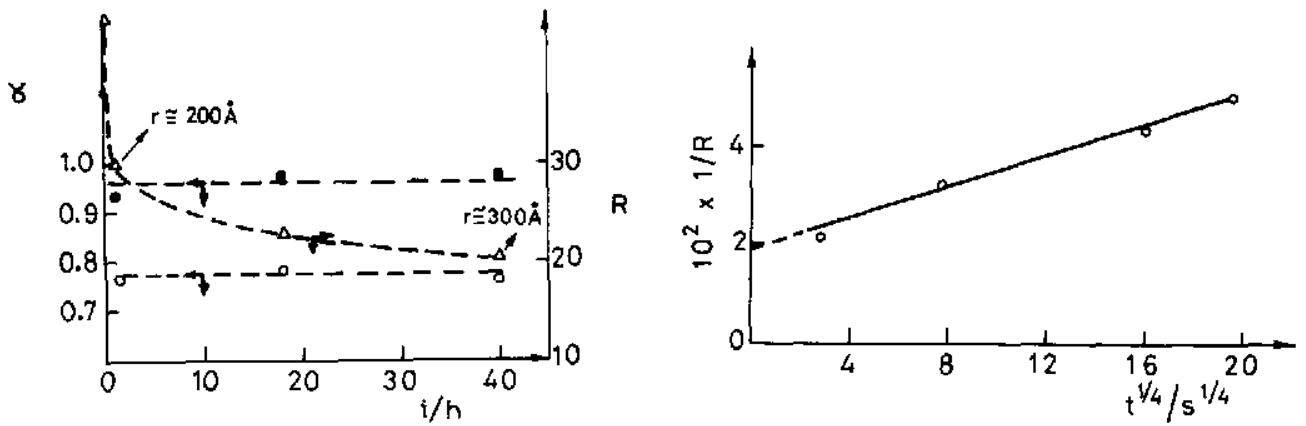

Fig. 6. Plots of $\alpha$ vs. $t$ obtained in $\mathrm{H}_{2} \mathrm{SO}_{4}(\bullet) 1 \mathrm{M}$ and (०) $1 \times 10^{-3} \mathrm{M}$ and $(\Delta)$ plot of $R$ vs. $t$. Average columnar radii at various times are indicated.

Fig. 7. Plot of $1 / R$ vs $t^{1 / 4}$ for data shown in Fig. 5.

decreased from 45 to 20 , the value of $\alpha$ measured in $10^{-3} \mathrm{M} \mathrm{H}_{2} \mathrm{SO}_{4}$ remains independent of the ageing time $t$.

\section{DISCUSSION}

The interpretation of the impedance spectra of EDAuE's in contact with different electrolyte acid solutions can be based on the facts that the surface chemistry and the structure have been already reasonably established. Thus, the electrochemical behaviour of gold in the potential range where the impedance measurements were made approaches the ideal conditions of a blocking electrode, i.e. an ideally polarizable electrode, independent of the $R$ value. Besides, the structure can be unambigously described as a columnar thin metal film. For such a system the value of $R$ has been related to the average geometrical parameters of the deposit by the simple equation [17]:

$R \equiv \pi h / 3 r$

where $h$ is the average height (which corresponds to the metal film thickness) and $r$ is the average radius of columns. According to a preceding work [17,19], linear $R$ vs. $h$ relationships have been obtained for over two decades in the magnitude of the $h$ scale with a slope $b=\pi / 3 r$. This means that the average value of $r$ appears as a constant, $r \equiv 1 \times 10^{-6} \mathrm{~cm}$ for the range of $h$ covered by the present work. For this simple columnar structure, the pore models $[5,20]$ could be implicated in the origin of the frequency dispersion observed for $R>20$ and $c<10^{-2} \mathrm{M}\left(\rho>300 \Omega \mathrm{cm}^{-1}\right)$. The cylindrical pore model has been applied to study the structure of various powder catalysts $(5,10)$. For this model, the current penetration, $\lambda$, into a pore of length $h$ is given by

$\lambda=1 / 2\left(r_{\mathrm{p}} / C_{\mathrm{d}} \rho \omega\right)^{1 / 2}$ 
where $r_{\mathrm{p}}$ is the pore radius, $C_{\mathrm{d}}$ is the specific capacitance and $\rho$ is the electrolyte resistivity. By using eqn. (3) and $C_{\mathrm{d}}=2 \times 10^{-5} \mathrm{~F} \mathrm{~cm}^{-2}, \rho=2 \times 10^{3} \Omega \mathrm{cm}^{-1}$ $\left(1 \times 10^{-3} \mathrm{M}\right)$ for $r_{\mathrm{p}}=1 \times 10^{-4} \mathrm{~cm}$ (the biggest pores) $\lambda$ becomes $1 \times 10^{-4} \mathrm{~cm}$ and $1 \times 10^{-3} \mathrm{~cm}$ at $f=10 \mathrm{KHz}$ and $f=100 \mathrm{~Hz}$, respectively. Considering that $h$ ranges between $3 \times 10^{-5} \mathrm{~cm}$ and $2 \times 10^{-4} \mathrm{~cm}$, these values imply that the largest pores are totally penetrated by the current. Conversely, for $r_{\mathrm{p}}=1 \times 10^{-6} \mathrm{~cm}$ (the smallest pores) one obtains $\lambda=1 \times 10^{-5} \mathrm{~cm}$ and $\lambda=1 \times 10^{-4} \mathrm{~cm}$ for $f=10 \mathrm{KHz}$ and $f=100 \mathrm{~Hz}$, respectively. This means that at the higher frequencies the small pores are only partially penetrated by the current so that their surfaces contribute partially to the sampled electrode area. These small pores appear to be responsible for the observed frequency dispersion. These considerations take into account the absence of marked changes in $\alpha$ during recrystallization. As was mentioned before, the ageing process influences the value of $R$ as the columnar structure goes in the direction of producing an increase of $r$ at a nearly constant $h$. It has been recently found that the kinetic law for roughness decay at EDAuE's and EDPtE's is given by the equation [21]:

$R^{-1}=B t^{1 / 4}+K$

where $B=2\left(\gamma a^{4} \mathbf{D}_{\mathrm{s}} / \mathbf{k T}\right)^{1 / 4}(3 / \pi h)$ and $K=r_{0} / h$. In eqn. (4) $\gamma$ is the surface tension of the gold, $a$ is the lattice parameter, $D_{\mathrm{s}}$ is the surface diffusion coefficient of atoms at the absolute temperature $T, k$ is the Boltzmann constant, and $r_{0}$ is the $r$ value for $t=0$. From the slope of the straight line resulting by plotting $R^{-1}$ vs $t^{1 / 4}$ (Fig. 7), and by using $\gamma=900 \mathrm{erg} / \mathrm{cm}^{2}, a=4.07 \times 10^{-\mathrm{B}} \mathrm{cm}, T=288 \mathrm{~K}$ and $h=1 \times 10^{-5} \mathrm{~cm}$, one obtains $D_{\mathrm{s}}=1 \times 10^{-14} \mathrm{~cm}^{2} / \mathrm{s}$. Likewise, from the value of $K$, $r_{0}=10^{-6} \mathrm{~cm}$ results. Both figures are in good agreement with data derived previously throughout the evolution of the rough gold surface $[21,22]$. Therefore, the validity of equation (4) allows the values of $r$ for the aged, rough structure at various ageing times to be estimated (Fig. 6). In this case the progressive increase in the grain size, which leads to an increase in $r_{\mathrm{p}}$ produces only a very slight change in the value of $\alpha$ for the frequency range studied in this work. This experimental fact can be explained considering the changes introduced in the film structure by the reconstruction process. The latter involves mass transfer between the tops of the columnar elements forming, by coalescence of small units of $20-30 \mathrm{~nm}$ in size, bigger ones of 50-60 nm without any marked change in film thickness $(h)$. Thus, small pores grow at a practically constant $h$ producing a large decrease in roughness ( $R$ proportional to $1 / r$ ) (Fig. 6 ). Thus, the shape of the resulting pore is maintained as its length differs slightly and the change in size is relatively small ( $r_{\mathrm{p}} \ll$ pore length $\equiv h$ ). This means that $\alpha$ cannot serve as an indicator of the changes produced during recrystallization.

However, the cylindrical pore model requires $\alpha=0.5$ rather than $\alpha \equiv 0.7$ observed in these systems. It is evident that a columnar model involving values of $r$ which are independent of the film thickness has been considered only as a rough approximation to real columnar systems. The STM measurements reveal that $r$ and $r_{\mathrm{p}}$ increase with $h$ as expected from theoretical considerations [23]. The change in $r_{\mathrm{p}}$ 
with $h$ should explain the slight increase in the $\mathrm{C}$ values observed in the dilute solution when $R$ changes from 30 to 135 (Fig. 5). Then, one should use the pore models developed in ref. 20. For different pore geometries, i.e. horn-shaped pores, if the decrease in pore radius is slower with pore depth, the value of $\alpha$ becomes smaller and approaches the limit $\alpha=0.5$. Using these models $\alpha$ values between 0.75 and 0.66 could be explained. However, a close inspection of Figs. 1 and 2 suggests the fractal nature of the electrode surface. So, in principle, fractal models seem to be applicable. According to these models the frequency dispersion is due to the fractal nature of the electrode/electrolyte interface and the frequency exponent $\alpha$ is related to the fractal dimension $D$ of the electrode surface. In general, two different models can be used; their validity can be tested by estimating $D$ from $\alpha$ and comparing it with the $D$ value measured by an independent method. The first model introduced by Liu [9] for a self-affine fractal surface gives a linear relationship between $D$ and $\alpha[11]$

$D=3-\alpha$

Taking $\alpha=0.74$ for our results at $3 \times 10^{-5}<h<3 \times 10^{-4}$ and $\rho>300 \Omega \mathrm{cm}^{-1}, D$ is 2.26. This value is lower than $D=2.5 \pm 0.1$ obtained for the same $h$ range by using an island type analysis of the ramified voids by STM [24] which applies to both self-affine and self-similar surface fractals. It should be noted that when Liu's model is generalized there is no universal relation in which $\alpha$ is a simple function of $D(25)$.

The second model proposed by Le Méhaute (1) for self-similar fractals gives a reciprocal relationship between $\mathrm{D}$ and $\alpha$ of the type

$D=1 / \alpha+1$

For electrochemically grown silver deposits a better agreement was observed using eqn. (6) rather than (5) [11]. However, in this case, for $\alpha=0.74$, one obtains $D=2.35$ which is also lower than $D=2.5 \pm 0.1$ obtained in ref. 24. As the differences between the $D$ values derived from the STM method and those obtained from the two models are similar, one cannot discriminate with confidence between these models.

Nevertheless, it was recently reported that a self-similar surface fractal does not show the behaviour described by eqn. (1) [18]. For self-similar surface fractals only a transition from the low frequency capacitive behaviour to the high frequency resistive behaviour over a small range of frequencies is observed [18]. It should be noted that the assertion that all fractal interfaces have a fractional-power-law impedance has been questioned (26). Conversely, self-affine surfaces exhibit a power-law scaling of the effective impedance with $D=3-\alpha(18)$.

Thus, from the present results one can only conclude that the columnar gold surface behaves as a self-affine surface fractal as it exhibits self similar behaviour of the impedance (eqn. (1)). This conclusion is supported by the STM-SEM data (Figs. 1 and 2) and also from large scale computer simulations of columnar structures $[14,27]$. The origin of the frequency dispersion can be explained qualita- 
tively considering that as $\omega$ increases the contribution of the smallest pores to the electrode area decreases, leading to a complicated surface impedance. Finally, it should be noted that some dependence of $\alpha$ on $\rho$ can be deduced from the experimental results (Table 1). This behaviour was reported for rough metal electrodes in different electrolytes [12]. This suggests the possibility that the electrolyte side of the interface also plays a role in determining the $\alpha$ value of the system.

\section{ACKNOWLEDGEMENTS}

Financial support was obtained through projects PS88-0014 (DGICYT) and MAT89-0204 (CICYT). RCS thanks CICYT (Spain) and CONICET (Argentina) for Fellowship grants.

\section{REFERENCES}

1 A. Le Méhaute and G. Crépy, Solid State Ionics, 9-10 (1983) 17.

2 B. Sapoval, J.N. Chazaviel and J. Peyrière, Solid State Ionics, 28-30 (1988) 1491.

3 J.C. Wang and J.B. Bates, Solid State Ionics, 18-19 (1986) 224.

4 R. de Levie, J, Electroanal. Chem., 281 (1990) 1.

5 R. de Levie, Electrochim. Acta, 10 (1965) 113.

6 L. Nyikos and T. Pajkossy, Electrochim. Acta, 30 (1985) 1533.

7 T.C. Halsey, Phys. Rev. A, 35 (1987) 3512.

8 R. Ball and M. Blunt, J. Phys. A, 21 (1988) 197.

9 H. Liu, Phys. Rev. Lett., 55 (1985) 529.

10 J.P. Candy, P. Fouilloux, M. Keddam and H. Takenouti, Electrochim. Acta, 26 (1981) 1029.

11 G.L.M.K.S. Kahanda and M. Tomkiewicz, J. Electrochem. Soc., 137 (1990) 3423.

12 E. Chassaing, B. Sapoval, G. Daccord and R. Lenormand, J. Electroanal. Chem., 279 (1990) 67; and references therein.

13 J.E. Yehoda and R. Messier. Appl. Surf. Sci., 22/23 (1985) 590.

14 P. Meakin, P. Ramanlal, L.M. Sander and R.C. Ball, Phys. Rev. A, 34 (1986) 591.

15 A.C. Chialvo, W.E. Triaca and A.J. Arvia, J. Electroanal. Chem., 146 (1983) 93.

16 L. Vázquez, J. Gómez, A.M. Baró, N. García, M.L. Marcos, J. González-Velazco, J.M. Vara and A.J. Arvia, J. Am. Chem. Soc., 109 (1987) 1730.

17 L. Vázquez, A. Bartolomé, A.M. Baró, C. Alonso, R.C. Salvarezza and A.J. Arvia, Surf. Sci., 215 (1989) 171.

18 D. Koch and A. Sangani, J. Electrochem. Soc., 138 (1991) 475.

19 R.C. Salvarezza, C. Alonso, J.M. Vara and A.J. Arvia, Phys. Rev. B., 41 (1990) 12502.

20 J.C. Wang and J.B. Bates, Solid State lonics, 18/19 (1986) 24.

21 C. Alonso, R.C. Salvarezza, J.M. Vara, A.J. Arvia, L. Vázquez, A. Bartolomé and A.M. Baró, J. Electrochem. Soc., 137 (1990) 2161.

22 C. Alonso, R.C. Salvarezza, J.M. Vara and A.J. Arvia, Electrochim. Acta, 35 (1990) 1331.

23 C. Tang, S. Alexander and R. Bruisma, Phys. Rev. Lett., 64 (1990) 777.

24 J.M. Gómez-Rodriguez, A.M. Baró, L. Vázquez, R.C. Salvarezza, J.M. Vara and A.J. Arvia, J. Phys. Chem., in press.

25 T. Kaplan, L.J. Gray and S.H. Liu, Phys. Rev. B, 35 (1987) 5379.

26 J.C. Wang, Solid State Ionics, 28-30 (1988) 1436.

27 F. Family and T. Vicsek, J. Phys. A, 18 (1985) L75. 